\title{
A New Method of Command and Guide the Unbalanced Distribution of Target
}

\author{
GAO Yangyang, YU Minjian, YOU Hanghang and JI Huiming \\ Air Force Engineering University, Air Traffic Control and Navigation College, 710051 Xi'an, China
}

\begin{abstract}
In the process of air combat implementation, the real-time command and guidance of air pilot is the key element of air combat irreplaceable. In this paper, a multi-aircraft air combat command and guidance target allocation model is established based on the actual air combat situation. The unbalanced target allocation problem of multiaircraft air combat command and guidance is studied by combining Hungarian algorithm and evolutionary Hungarian algorithm. Finally, two cases of increasing aircraft number and decreasing aircraft number are simulated and studied. Simulation results show that the method can shorten the computation time and ensure the overall command and guidance advantages of the target allocation scheme effectively.
\end{abstract}

\section{Introduction}

In the networked modern air combat of multi-aircraft cooperative confrontation, the guidance and allocation of enemy and fighter planes is an important link in the command and guidance of multi-aircraft air combat. Combine with that concrete reality of our army's command and guidance allocation problem, we mainly carry out one-to-one or many-to-one task allocation, so we can treat the command and guidance allocation problem as a target allocation problem.

Literature[1][2] has studied the target assignment of the United States air force unmanned aerial vehicle ( UAV ) cooperative operation. The problem of UAV cooperative control and task assignment is studied in reference [3]. At the domestic level, the Taiwan university of science and technology has conducted research on the distribution of weapons targets [4]; In the mainland, there are also many scholars on the target allocation problem has been studied, Lv Hongwei, etc from the air combat geometry situation, put forward to our overall geometric advantage of the largest target allocation algorithm, so as to give full play to the overall formation advantage [5]. Chen Jieyu et al. proposed a target allocation algorithm based on distributed auction mechanism, which can get the optimization scheme quickly [6]. [7] considering the advantages of simulated annealing algorithm, such as strong robustness, good universality and easy implementation, the simulated annealing algorithm is used to solve the weapon-target assignment problem.

Based on the practical consideration of multi-aircraft air combat, this paper divides the command and guidance assignment problem into small-scale assignment problem and large-scale assignment problem. Hungary algorithm and evolutionary Hungary algorithm are used for target assignment respectively, which not only ensures the accuracy of the assignment results, but also shortens the operation time.

\section{Target assignment model}

\subsection{Concept of target assignment}

Considering the number of aircraft, target allocation has two ways, one is balanced target allocation, the other is unbalanced target allocation.

\subsubsection{Balanced target distribution}

Balanced target distribution method refers to the air combat, both sides forces are consistent, and aircraft ability is equal. According to the battlefield situation, the enemy and our aircraft to " one - to - one" distribution mode, balanced distribution method is generally the original distribution method.

\subsubsection{Unbalanced target distribution}

In actual operations, the problem of balanced target allocation is only a relatively ideal situation. Usually, the number of aircraft on both sides is not equal. Unbalanced target allocation method is more complex than balanced target allocation method. During processing, the number of targets can be balanced by adding virtual targets, which can be transformed into balanced target allocation problem. 


\subsection{Multi - aircraft air combat command and guidance target assignment model}

In multi-aircraft air combat command and guidance target allocation, only the number of enemy and our aircraft in air combat needs to be considered, so as to model the command and guidance target allocation. Suppose there are $m$ enemy planes and $n$ our planes in air combat, each of which can attack only one enemy plane at the same time. Our fighter plane is composed of cluster $W=\left\{W_{i}, i=1,2, \cdots, n\right\}$ and enemy target machine is composed of cluster $D=\left\{D_{j}, j=1,2, \cdots, m\right\}$. Among them, $W_{i}$ represents our $i t h$ fighter, $D_{j}$ represents the enemy's $j$ th fighter, in order to achieve air combat victory, usually in the use of forces against the enemy forces constitute an advantage, so should meet $n \geq m$, the multi-aircraft air combat command guidance target allocation model can be described as follows:

$$
\begin{gathered}
z=\max \sum_{i=1}^{n} \sum_{j=1}^{m} C_{i j} X_{i j} \\
\sum_{i=1}^{n} X_{i j}=1, j=1, \cdots, m \\
\sum_{j=1}^{m} X_{i j}=1 \text { or } 2, \quad i=1,2, \cdots, n
\end{gathered}
$$

Satisfy:

$$
X(i, j)=\left\{\begin{array}{l}
1 \\
0
\end{array}\right.
$$

Among them, $C=\left(C_{i j}\right)_{n \times m}$ is the evaluation matrix, and $C_{i j}$ is the air combat command and guidance dominance value of the ith aircraft to the $j$ th enemy aircraft. $X=\left(X_{i j}\right)_{n \times m}$ is the solution matrix of target distribution, $X_{i j}$ value of 1 said has been assigned the $j$ th enemy aircraft to the ith aircraft, value of 0 , said not to assign the $j$ th enemy aircraft to the ith aircraft. $Z$ is the objective function, said our fleet total command guidance advantage value, reflect our fleet total air combat situation advantage. An assignment solution $X$ is given to maximize the commander-in-chief lead advantage value of $Z$. Constraints (2) and (3) respectively indicate that one of our aircraft can attack only one enemy aircraft and one enemy aircraft can be attacked by two of our aircraft at the same time, reflecting the characteristics of multi-aircraft air combat command and guidance target allocation.

\section{Multi - objective task assignment based on Hungarian algorithm}

\subsection{Hungarian algorithm}

Hungarian algorithm was proposed by Kuhn in 1955. it is a solution to assignment problem.

The general implementation steps of the Hungarian algorithm are as follows:
Step 1: Randomly number enemy and our fighter.

Step 2: Based on the attribute of enemy aircraft and the air combat situation of enemy and our aircraft, the situation assessment is carried out, and then the evaluation matrix is constructed.

Step 3: Linearly transform the evaluation matrix so that 0 elements appear in each column of each row. (Each line and column minus the smallest element).

Step 4: To obtain the optimal allocation result from the adjusted evaluation matrix.

\subsection{Evolutionary Hungarian algorithm}

Evolutionary Hungarian algorithm is proposed by Holland. Evolutionary algorithm can effectively solve the problem of target allocation, but the computational speed decreases significantly with the increase of dimension, which cannot meet the real-time requirements of battlefield. In this paper, we combine Hungarian algorithm with evolutionary Hungary to deal with largescale target allocation. The algorithm flow is as follows:

Step 1: Judge the number of the fighter planes on both sides and randomly number the fighter planes. If the number of aircraft on both sides is not more than 4 , can be directly calculated using simple Hungarian algorithm. If the number of aircraft is more than 4 , it is considered as a large-scale target allocation problem, and the two algorithms are combined to deal with it.

Step 2: In dealing with large-scale target allocation problem, the evaluation matrix is firstly processed by Hungarian algorithm to obtain corresponding solution matrix, then several corresponding $0-1$ matrices are randomly generated, and these $0-1$ matrices and the obtained solution matrix are taken as the initial population of the algorithm.

Step 3: The fitness of each individual in the population is calculated, and replication and variation are carried out.

Step 4: Increasing the evolution algebra until the maximum evolution algebra is reached, and then selecting the individual in the obtained population, wherein the optimal individual is the required solution, and the corresponding target distribution result is the optimal distribution scheme.

\subsection{Processing of dynamic target assignment}

In air combat, the forces of both sides will change due to the damage or withdrawal of aircraft, so the target allocation will be a dynamic process.

\subsubsection{The situation of aircraft out of the battlefield}

When a fighter exits the battlefield, the rows or columns of the corresponding evaluation matrix should be deleted. however, in order to maintain the integrity of the evaluation matrix and enable the evaluation matrix to be used in the calculation and processing of the algorithm, according to the margin method proposed in this paper, the row or column element value of the corresponding evaluation matrix of the fighter exiting the battlefield is 
changed to 0 , which not only ensures the integrity of the evaluation matrix, but also ensures that the changed element value does not affect the normal target allocation.

\subsubsection{The situation of aircraft into the battlefield}

When a fighter plane is newly added to a battlefield, the number of the fighter planes in the battlefield changes, and the evaluation matrix also changes accordingly, and the number of rows and columns of the evaluation matrix is not equal at this time, and the edge mending method is adopted for processing.

\section{Simulation analysis}

\subsection{Increased number of aircraft}

In air combat, there are three kinds of initial situations of the number of fighter planes on both sides: the number of our aircraft is dominant, the number of enemy aircraft is dominant and the number of both sides is equal. But in actual operations, in order to ensure the operational effect, often won't appear under the initial situation of our number is less than the number of enemy aircraft, our number is dominant, the number of enemy aircraft after increase can be converted into a balanced target allocation problem, in this paper, we discuss the number of both sides is equal. Assume that the corresponding evaluation matrix is $T_{4 \times 4}$.

$$
T_{4 \times 4}=\left[\begin{array}{cccc}
245 & 345 & 578 & 335 \\
567 & 680 & 123 & 280 \\
467 & 134 & 232 & 167 \\
225 & 115 & 245 & 496
\end{array}\right]
$$

The Hungarian algorithm is use for calculation and processing, and that result matrix is as follow:

$$
T_{4 \times 4}=\left[\begin{array}{cccc}
0 & 0 & 1 & 0 \\
0 & 1 & 0 & 0 \\
1 & 0 & 0 & 0 \\
0 & 0 & 0 & 1
\end{array}\right]
$$

The corresponding allocation results are as follows: our aircraft No.1 attacked enemy aircraft No.3; Our aircraft No. 2 attack enemy aircraft No. 2; Our aircraft No. 3 attack enemy aircraft No. 1; Our aircraft No. 4 attacked enemy aircraft No. 4.

If suddenly increase aircraft, according to the edge method to change the evaluation matrix. In this initial condition, if the enemy add a fighter, through the analysis of the battlefield situation, get the new fighter threat value, the corresponding after the evaluation matrix to add a column of the aircraft threat value, at the same time need to add the corresponding row below the evaluation matrix for " edge", the principle is that does not affect the normal target distribution. The modified evaluation matrix is:

$$
T_{5 \times 5}=\left[\begin{array}{lllll}
245 & 345 & 578 & 335 & 99 \\
567 & 680 & 123 & 280 & 322 \\
467 & 134 & 232 & 167 & 431 \\
225 & 115 & 245 & 496 & 506 \\
0 & 0 & 0 & 0 & 0
\end{array}\right]
$$

The result matrix is:

$$
T_{5 \times 5}=\left[\begin{array}{ccccc}
0 & 0 & 1 & 0 & 0 \\
0 & 1 & 0 & 0 & 0 \\
1 & 0 & 0 & 0 & 0 \\
0 & 0 & 0 & 0 & 1 \\
0 & 0 & 0 & 1 & 0
\end{array}\right]
$$

The corresponding allocation results are as follows: our aircraft No.1 attacked enemy aircraft No.3; Our aircraft No. 2 attack enemy aircraft No. 2; Our aircraft No. 3 attack enemy aircraft No. 1; Our aircraft No. 4 attack enemy aircraft No. 5; Our aircraft No. 5 attack enemy aircraft No. 4. As shown in figure 1:

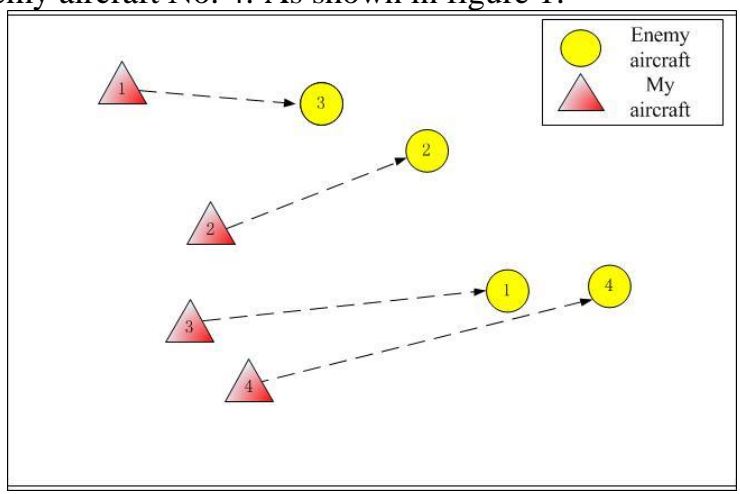

Figure 1. The distribution after enemy joining

\subsection{Reduced number of aircraft}

In the discussion of the above initial conditions, it is assumed that the corresponding evaluation matrix is $T_{4 \times 4}$.

$$
T_{4 \times 4}=\left[\begin{array}{cccc}
245 & 345 & 578 & 335 \\
567 & 680 & 123 & 280 \\
467 & 134 & 232 & 167 \\
225 & 115 & 245 & 496
\end{array}\right]
$$

The result matrix is:

$$
T_{4 \times 4}=\left[\begin{array}{cccc}
0 & 0 & 1 & 0 \\
0 & 1 & 0 & 0 \\
1 & 0 & 0 & 0 \\
0 & 0 & 0 & 1
\end{array}\right]
$$

The corresponding allocation results are as follows: our aircraft No.1 attacked enemy aircraft No.3; Our aircraft No. 2 attack enemy aircraft No. 2; Our aircraft No. 3 attack enemy aircraft No. 1; Our aircraft No. 4 attacked enemy aircraft No. 4.

When the enemy aircraft No. 3 out of the battlefield, using the edge method need to modify the third column is as follows: 


$$
T_{4 \times 4}^{\prime}=\left[\begin{array}{cccc}
245 & 345 & 0 & 335 \\
567 & 680 & 0 & 280 \\
467 & 134 & 0 & 167 \\
225 & 115 & 0 & 496
\end{array}\right]
$$

The result matrix is:

$$
T_{4 \times 4}^{\prime}=\left[\begin{array}{cccc}
0 & 0 & 1 & 0 \\
0 & 1 & 0 & 0 \\
1 & 0 & 0 & 0 \\
0 & 0 & 0 & 1
\end{array}\right]
$$

The corresponding allocation results are as follows: our aircraft No.1 attacked enemy aircraft No.3; Our aircraft No. 2 attack enemy aircraft No. 2; Our aircraft No. 3 attack enemy aircraft No. 1; Our aircraft No. 4 attacked enemy aircraft No. 4.

As shown in figure 2:

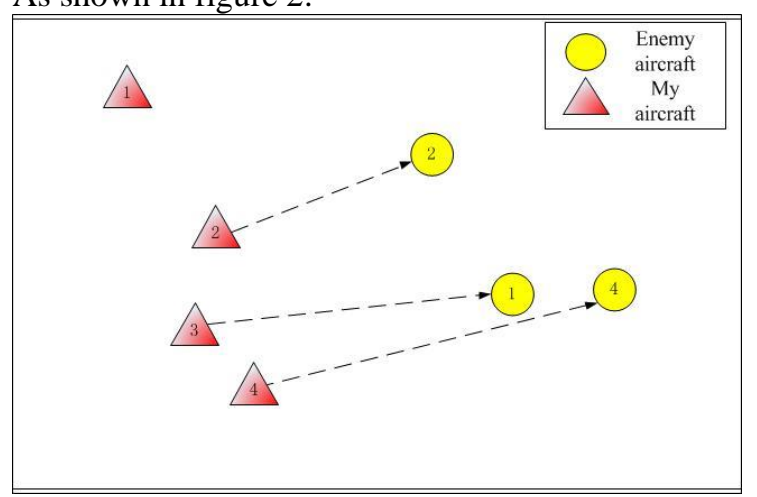

Figure 2. The distribution of enemy forces after withdrawal

In the case of Figure 2, you can choose to match the aircraft No. 2 attack on enemy aircraft No. 2 with my aircraft No.1, and the redistribution is shown in Figure 3.

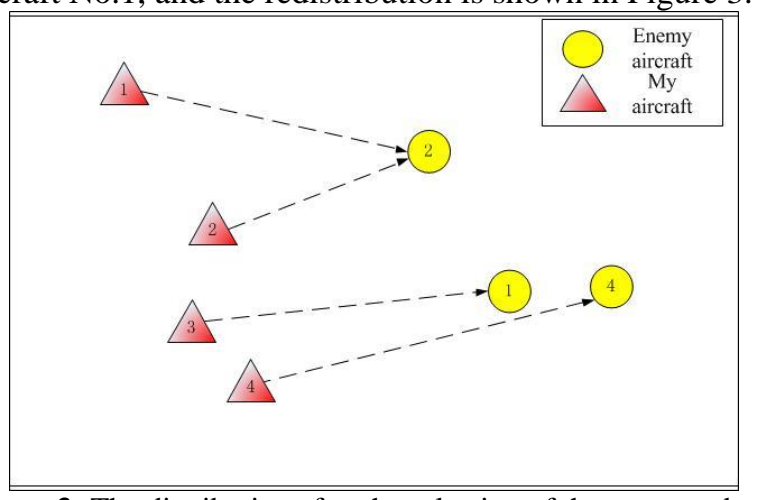

Figure 3. The distribution after the selection of the commander

\section{Conclusion}

Based on Hungarian algorithm, this paper studies the problem of command and guidance target assignment. Hungary algorithm is used to discuss the unbalanced target allocation method and the dynamic reallocation method after the forces of both sides withdraw from the battlefield under the condition of the battlefield, and the simulation results are given. The overall command and guidance advantages of the target allocation scheme are effectively ensured.

\section{References}

1. KINGSTON D B, RASMUSSEN S J, MEARS M J. Base defense using a task assignment framework [C] / /AIAA Guidance, Navigation and Control Conference, Chicago, Illinois, doi: 10.2514/6.20096209.

2. ANDERSSON K, KAMINER I, JONES K D, et al. Cooperating UAVs using thermal lift to extend endurance [C ] / /AIAA Infotech @ Aerospace Conference, Washington doi:10.2514 /6. 20092043.

3. NIGAM N, KROO I. Control and design of multiple unmanned air vehicles for a persistent surveillance task [C] / /12th AIAA /ISSMO Multidisciplinary Analysis and Optimization Conference, Victoria, British Columbia Canada doi:10. 2514/6. 20085913.

4. LEE Z J, SU S F, LEE C Y. Efficiently solving general weapon-target assignment problem by genetic algorithms with greedy eugenics [J] . IEEE Transactions on Systems, Man, and Cybernetics, Part B: Cybernetics, 2003,33( 1) :113121.

5. LI X M, LI J F,et al. Method of Static Precision Corrected of Carrier Radar Based on CCD-LaserTheodolite[J]. Journal of Naval Aeronautical and Astronautical University,2008,23(1): 59-61.

6. Wu P, Liang Q. Simulated Annealing Algorithm for Weapon- Target Assignment Problem[J].Computer \& Digital Engineering, 2013,41(2):201-203.

7. CHEN J ,YAO P Y. Multi-UAV Decentral ized Coopertative Dynamic Target Assignment Method[J]. Journal of Air Force Engineering University,2014,15(6):11-16.

8. WANG Z,LIU Z Y, PEI R,et al. State Estimation For Uncertain System By Moving Horizon Strategy[J]. Proceedings of the CSEE,2003,23(9):99-102.

9. ZHAO H Y, GAO X Q,C H.Application of moving horizon estmiation in three tank system[J].Journal of Jilin University (Information Science Edition), 2004,22 (4): 298- 302.

10. KANG L, ZHAO C X, GUO J H. Path Planning Based on Fuzzy Rolling Rapidly exploring Random Tree for Mobile Robot[J].Journal of Nan jing University of Science and Technology (Natural Science),2010,35(5):642-648.

11. HUO A Q,DAI C,WANG Y L, et al. Predictive control in stabilized platform based on augmented non-minimalstate spacemethod.ComputerEngineeringandApplications, 2013,49,(17):239-244. 